PROCEEDINGS OF THE

AMERICAN MATHEMATICAL SOCIETY

Volume 132, Number 12, Pages 3589-3597

S 0002-9939(04)07434-9

Article electronically published on July 22, 2004

\title{
CONVERGENCE ESTIMATES OF GALERKIN-WAVELET SOLUTIONS TO A CAUCHY PROBLEM FOR A CLASS OF PERIODIC PSEUDODIFFERENTIAL EQUATIONS
}

\author{
NGUYEN MINH CHUONG AND BUI KIEN CUONG \\ (Communicated by David R. Larson)
}

\begin{abstract}
A class of Cauchy problems for interesting complicated periodic pseudodifferential equations is considered. By the Galerkin-wavelet method and with weak solutions one can find sufficient conditions to establish convergence estimates of weak Galerkin-wavelet solutions to a Cauchy problem for this class of equations.
\end{abstract}

\section{INTRODUCTION}

The theory of pseudodifferential operators has been extremely intensively developing. This is so, not only because it is a very general theory including many theories, especially because it is of great interest and difficulty, but also because it is an extremely powerful tool for studying linear and nonlinear problems (see [3], [5]-77, [11, 12], [16, 19], 21], [22] and references therein). Wavelet theory is growing very rapidly as well (see [1]-[4, 8]-[15], [17], [18], 20] and references therein). Wavelet approximation methods, however, for even only pseudodifferential operators (not a Cauchy problem) are investigated in a very few works [2], 10], [13], [14, [18].

In this paper we study approximately a Cauchy problem for a class of interesting complicated pseudodifferential equations as follows:

$$
\begin{aligned}
& \frac{\partial u}{\partial t}=-\mu A u(x, t)-\int_{0}^{t} a(t-\tau) A u(x, \tau) d \tau+b(x, t), \\
& u(x, 0)=u_{0}(x)
\end{aligned}
$$

where $x \in \mathcal{J}^{n}=\mathbb{R}^{n} / \mathbb{Z}^{n}, \mu>0, A=\sigma(D)$ is a pseudodifferential operator, defined by

$$
\sigma(D) u(x)=\sum_{\xi \in \mathbb{Z}^{n}} e^{2 \pi i x \xi} \sigma(\xi) \tilde{u}(\xi), u \in C^{\infty}\left(\mathbb{Z}^{n}\right),
$$

with symbol $\sigma(\xi)$ belonging to $S^{2 m}\left(\mathbb{Z}^{n}\right)$, i.e. $\sigma \in C^{\infty}\left(\mathbb{Z}^{n}\right)$ satisfying

$$
\left|\Delta^{\alpha} \sigma(\xi)\right| \leq C_{\alpha}(1+|\xi|)^{2 m-|\alpha|} \text { for all } \xi \in \mathbb{Z}^{n} \text { and for all multi-indexes } \alpha,
$$

Received by the editors November 15, 2001 and, in revised form, June 20, 2003.

2000 Mathematics Subject Classification. Primary 35A40,65D05, 65M06, 65M12, 65M15.

Key words and phrases. Convergence estimates, weak Galerkin-wavelet solution, Cauchy problem, periodic pseudodifferential equations.

(C)2004 American Mathematical Society 
and $\Delta$ is the difference operator, which is defined by $\Delta:=\left(\tau_{1}-1, \tau_{2}-1, \ldots, \tau_{n}-1\right)^{T}$, where $\tau_{j} f(x):=f\left(x+e^{j}\right), e^{j}=\left(\delta_{j, l}\right)_{l=1}^{n}$ is the $j$ th coordinate vector.

Here it is assumed also that $\sigma \in C^{\infty}\left(\mathbb{R}^{n} \backslash\{0\}\right)$ and $\sigma(t \xi)=t^{2 m} \sigma(\xi), t>0, \sigma(0)=$ 1. Moreover

$$
\sigma(\xi) \geq c\left(1+|\xi|^{2}\right)^{m},|\xi| \geq R>0 .
$$

The functions $a(t), b(x, t)$ are given. Note that when $\mu=0$, under some assumptions on $a(t)$ the type of equation (1.1) may be changed.

To establish some convergence estimates for this complicated problem, in order to overcome the difficulties, let us use the Galerkin-wavelet method and weak solutions and approximative weak solutions, with the very effective tools: the Fourier and Laplace transforms.

\section{NotAtion AND PRELIMINARIES}

Let us recall here some usual notation, definitions and some basic facts.

For a function $u(x) \in L_{2}\left(\mathcal{J}^{n}\right)$, the discrete Fourier transform is defined by

$$
\mathcal{F}(u)(\xi)=\tilde{u}(\xi):=\int_{\mathcal{J}^{n}} e^{-2 \pi i x \xi} u(x) d x=\int_{[0,1]^{n}} e^{-2 \pi i x \xi} u(x) d x, \xi \in \mathbb{Z}^{n}
$$

The discrete inverse Fourier transform is then $u(x):=\sum_{\xi \in \mathbb{Z}^{n}} \tilde{u}(\xi) e^{2 \pi i x \xi}$.

The Fourier transform of the function $f \in L_{2}\left(\mathbb{R}^{n}\right)$ is

$$
\hat{f}(\xi)=\int_{\mathbb{R}^{n}} e^{-2 \pi i x \xi} f(x) d x, \xi \in \mathbb{R}^{n}
$$

with the inverse Fourier transform $f(x)=\int_{\mathbb{R}^{n}} e^{2 \pi i x \xi} \hat{f}(\xi) d \xi, x \in \mathbb{R}^{n}$.

The Laplace transform of a function $u(x)$ increasing less than an exponent is $L(u)(s)=\check{u}(s):=\int_{0}^{\infty} e^{-2 \pi s t} u(t) d t, s \in \mathbb{C}$. Below will be listed some usual simple properties of the Laplace transform,

$$
L(a * u)(s)=L(a)(s) \cdot L(u)(s)
$$

where

$$
(a * u)(t)=\int_{0}^{t} a(t-\tau) u(\tau) d \tau
$$

$$
\begin{aligned}
& L\left(u^{(k)}\right)(s)=-u^{(k-1)}(0)-2 \pi s u^{(k-2)}(0)-\ldots-(2 \pi s)^{(k-1)} u(0)+(2 \pi s)^{k} \breve{u}(s), \\
& \quad \int_{-\infty}^{+\infty} \check{u}\left(s_{0}+i \delta\right) \overline{\check{v}\left(s_{0}+i \delta\right)} d \delta=\int_{0}^{+\infty} e^{-4 \pi s_{0} t} u(t) \overline{v(t)} d t .
\end{aligned}
$$

We consider now the space $H^{s}\left(\mathcal{J}^{n}\right)(s \in \mathbb{R})$ of functions $u \in \mathcal{D}^{\prime}\left(\mathcal{J}^{n}\right)$ (the L. Schwartz space of distributions), such that $\langle D\rangle^{s} u \in L_{2}\left(\mathcal{J}^{n}\right)$ and the norm

$$
\|u\|_{s}=\left(\sum_{\xi \in \mathbb{Z}^{n}}\langle\xi\rangle^{2 s}|\tilde{u}(\xi)|^{2}\right)^{1 / 2}
$$

is finite, where

$$
\langle\xi\rangle= \begin{cases}1 \quad \text { if } \quad & \xi=0 \\ |\xi| & \text { if } \quad \xi \neq 0\end{cases}
$$


The norm of $u \in H^{s}\left(\mathcal{J}^{n}\right)$ is defined by (2.4).

Definition 1. Let $L_{2}\left(H^{q, s_{0}}\right)\left(q \geq 0, s_{0}>0\right)$ be the space of all functions $u(x, t), x \in$ $\mathcal{J}^{n}, t \geq 0$, satisfying the following conditions: for each $0 \leq \alpha \leq q$,

i) $u(x, t) \in H^{\alpha}\left(\mathcal{J}^{n}\right) \forall t \in[0,+\infty)$.

ii) The following series converges:

$$
\sum_{\xi \in \mathbb{Z}^{n}}\langle\xi\rangle^{2 \alpha} \int_{0}^{\infty} e^{-4 \pi s_{0} t}|\tilde{u}(\xi, t)|^{2} d t .
$$

The norm of the function $u \in L_{2}\left(H^{q, s_{0}}\right)$ is defined by

$$
\|u\|_{L_{2}\left(H^{q, s_{0}}\right)}=\left(\sum_{\xi \in \mathbb{Z}^{n}}\langle\xi\rangle^{2 q} \int_{0}^{\infty} e^{-4 \pi s_{0} t}|\tilde{u}(\xi, t)|^{2} d t\right)^{1 / 2} .
$$

The following lemmas are needed in the sequel.

Lemma 1. If $u \in L_{2}\left(H^{0, s_{0}}\right)$, then

$$
L(\tilde{u}(\xi, .))(s)=\mathcal{F}(\check{u}(., s))(\xi) .
$$

Lemma 2. If $u \in L_{2}\left(H^{q, s_{0}}\right), q \geq 2 m$ and $v \in L_{2}\left(H^{0, s_{0}}\right)$, then

$$
\begin{gathered}
\int_{-\infty}^{+\infty}\left\|v\left(., s_{0}+i \delta\right)\right\|^{2} d \delta=\int_{0}^{\infty} e^{-4 \pi s_{0} t}\|v(., t)\|^{2} d t \\
\int_{-\infty}^{+\infty}(A \check{u}, \check{v})\left(s_{0}+i \delta\right) d \delta=\int_{0}^{\infty} e^{-4 \pi s_{0} t}(A u, v) d t .
\end{gathered}
$$

Proof. It is obvious that

$$
\begin{aligned}
\int_{-\infty}^{+\infty}\left\|\check{v}\left(., s_{0}+i \delta\right)\right\|^{2} d \delta & =\int_{-\infty}^{+\infty} \sum_{\xi \in \mathbb{Z}^{n}}\left|\tilde{\tilde{v}}\left(\xi, s_{0}+i \delta\right)\right|^{2} d \delta=\sum_{\xi \in \mathbb{Z}^{n}} \int_{-\infty}^{+\infty}\left|\tilde{\tilde{v}}\left(\xi, s_{0}+i \delta\right)\right|^{2} d \delta \\
& =\sum_{\xi \in \mathbb{Z}^{n}} \int_{0}^{\infty} e^{-4 \pi s_{0} t}|\tilde{v}(\xi, t)|^{2} d t=\int_{0}^{\infty} e^{-4 \pi s_{0} t}\|\tilde{v}(., t)\|^{2} d t,
\end{aligned}
$$

that is, we obtain (2.9).

Similarly we prove (2.10), taking into account that we can integrate an infinite sum in the integration of two sides of the equality

$$
(A \check{u}, \check{v})\left(s_{0}+i \delta\right)=\sum_{\xi \in \mathbb{Z}^{n}} \sigma(\xi) \check{\tilde{u}}\left(\xi, s_{0}+i \delta\right) \overline{\tilde{v}\left(\xi, s_{0}+i \delta\right)} .
$$

Lemma 3. Under assumption (1.3) we have

$$
(A u, u) \geq c\|u\|_{m}^{2}, \forall u \in H^{2 m}\left(\mathcal{J}^{n}\right)
$$

where $c$ is independent of $u \in H^{2 m}\left(\mathcal{J}^{n}\right)$.

Proof. From (1.3) and the fact that $\sigma(0)=1$, it follows that there exists a number $c>0$ independent of $\xi$ such that $\sigma(\xi) \geq c\langle\xi\rangle^{2 m}, \xi \in \mathbb{Z}^{n}$. The definition of $A$ then gives (2.11).

Next we introduce some notation and definitions on wavelets. 
Definition 2. A multiresolution approximation (M.R.A.) of $L_{2}\left(\mathbb{R}^{n}\right)$ is, by definition, an increasing sequence $V_{j}, j \in \mathbb{Z}$, of closed linear subspaces of $L_{2}\left(\mathbb{R}^{n}\right)$ with the following properties:

$$
\begin{gathered}
\ldots \subset V_{-1} \subset V_{0} \subset V_{1} \subset \ldots \subset V_{n} \subset \ldots ; \\
\bigcap_{j \in \mathbb{Z}} V_{j}=\{0\}, \overline{\bigcup_{j \in \mathbb{Z}} V_{j}}=L_{2}\left(\mathbb{R}^{n}\right) ;
\end{gathered}
$$

for all $f \in L_{2}\left(\mathbb{R}^{n}\right)$ and all $j \in \mathbb{Z}$,

$$
f(x) \in V_{j} \Leftrightarrow f(2 x) \in V_{j+1}
$$

for all $f \in L_{2}\left(\mathbb{R}^{n}\right)$ and $k \in \mathbb{Z}^{n}$,

$$
f(x) \in V_{0} \Leftrightarrow f(x-k) \in V_{0} .
$$

There exists a function, which is called a scaling function (S.F.) $\phi(x) \in V_{0}$, such that the sequence $\left\{\phi(x-k), k \in \mathbb{Z}^{n}\right\}$ is a Riesz basic of $V_{0}$.

An M.R.A. of $L_{2}\left(\mathbb{R}^{n}\right)$ is said to be $r$-regular $(r \in \mathbb{N})$ if the function $\phi$ is $r$-regular, that is, for each $m \in \mathbb{N}$ there exists $c_{m}$ such that for all multi-indexes $\alpha,|\alpha| \leq r$, the following condition holds:

$$
\left|D^{\alpha} \phi(x)\right| \leq c_{m}(1+|x|)^{-m}
$$

Let us denote

$$
\phi_{j k}(x)=2^{n j / 2} \phi\left(2^{j} x-k\right), k \in \mathbb{Z}^{n} .
$$

Obviously

$$
V_{j}=\overline{\operatorname{span}}\left\{\phi_{j k}(x), k \in \mathbb{Z}^{n}\right\} .
$$

The notation $[u](x)$ stands for the periodization operator of a function $u(x)$, that is, $[u](x)=\sum_{k \in \mathbb{Z}^{n}} u(x+k)$. Denote

$$
\phi_{j}^{k}(x):=\left[\phi_{j k}\right](x)=2^{\frac{n j}{2}} \sum_{l \in \mathbb{Z}^{n}} \phi\left(2^{j}(x+l)-k\right) .
$$

Similarly let us define an M.R.A. of $L_{2}\left(\mathcal{J}^{n}\right)$ as follows:

$$
\left[V_{j}\right]:=\overline{\operatorname{span}}\left\{\phi_{j}^{k}(x), k \in \mathbb{Z}^{n j}\right\}, j \geq 0,
$$

where $\mathbb{Z}^{n j}=\mathbb{Z}^{n} / 2^{j} \mathbb{Z}^{n}$. It is easy to check that

$$
\left[V_{0}\right] \subset\left[V_{1}\right] \subset \ldots \subset\left[V_{n}\right] \subset \ldots, \quad \overline{\bigcup_{j \geq 0}\left[V_{j}\right]}=L_{2}\left(\mathcal{J}^{n}\right) .
$$

Furthermore, if

$$
\left(\phi_{j k}, \phi_{j l}\right)=\delta_{k l}, k, l \in \mathbb{Z}^{n}
$$

then

$$
\left(\phi_{k}^{j}, \phi_{l}^{j}\right)=\delta_{k l}, k, l \in \mathbb{Z}^{n j}
$$

and $\operatorname{dim}\left[V_{j}\right]=2^{n j}$.

For each $j \geq 0$, let $P_{j}: L_{2}\left(\mathcal{J}^{n}\right) \rightarrow\left[V_{j}\right]$ be the orthogonal projection of $L_{2}\left(\mathcal{J}^{n}\right)$ on $\left[V_{j}\right]$, which has the property

Theorem 1 ([14]). Let $-r-1 \leq s \leq r,-r \leq q \leq r+1$ and $s \leq q$. Then

$$
\left\|u-P_{j} u\right\|_{s} \leq c 2^{j(s-q)}\|u\|_{q}
$$

for all $u \in H^{q}\left(\mathcal{J}^{n}\right)$, where $c$ is a constant independent of $j$ and $u$. 
By denoting $h=2^{-j}$ and $V_{h}=\left[V_{j}\right]$, we can write (2.12) as follows:

$$
\left\|u-P_{j} u\right\|_{s} \leq c h^{q-s}\|u\|_{q} .
$$

\section{Convergence estimates of solutions}

Definition 3. A $C^{1}$-mapping $u$ in $t, u:[0, \infty) \rightarrow H^{2 m}\left(\mathcal{J}^{n}\right)$ satisfying

$$
\begin{aligned}
& \left(\frac{\partial u}{\partial t}, v\right)=-\mu(A u, v)-\int_{0}^{t} a(t-\tau)(A u(\tau), v) d \tau+(b, v), \\
& (u(x, 0), v)=\left(u_{0}, v\right), \forall v \in L_{2}\left(\mathcal{J}^{n}\right)
\end{aligned}
$$

is called a weak solution of problem (1.1)-(1.2).

Definition 4. A $C^{1}$-mapping $u_{h}$ in $t, u_{h}:[0, \infty) \rightarrow V_{h}$ satisfying

$$
\begin{aligned}
& \left(\frac{\partial u_{h}}{\partial t}, v\right)=-\mu\left(A u_{h}, v\right)-\int_{0}^{t} a(t-\tau)(A u(\tau), v) d \tau+(b, v), \\
& \left(u_{h}(x, 0), v\right)=\left(u_{0 h}, v\right), \forall v \in V_{h},
\end{aligned}
$$

where $u_{0 h}:=\mathcal{R} u_{0}$ is a linear approximation of $u_{0}$ in $V_{h}$, is called a weak Galerkinwavelet solution of problem (1.1)-(1.2).

The following theorem asserts the stability of the weak solution of problem (1.1)(1.2).

Theorem 2. Let $u(x, t) \in L_{2}\left(H^{2 m, s_{0}}\right)$ be a weak solution of problem (1.1)-(1.2) with $b=0$. Assume, moreover, that there exists a number $s_{0}>0$ such that

$$
\mu+\operatorname{Re} \check{a}\left(s_{0}+i \delta\right) \geq 0, \forall \delta \in \mathbb{R} .
$$

Then

$$
\|u\|_{L_{2}\left(H^{\left.0, s_{0}\right)}\right.}^{2} \leq \frac{1}{4 \pi s_{0}}\left\|u_{0}\right\|^{2}
$$

Proof. In (3.1), choosing $v=u$, multiplying two sides with $e^{-4 \pi s_{0} t}$ and integrating in $t$ from 0 to $+\infty$ we obtain

$$
\begin{aligned}
\int_{0}^{+\infty} e^{-4 \pi s_{0} t}\left(\frac{\partial u}{\partial t}, u\right) d t= & -\mu \int_{0}^{\infty} e^{-4 \pi s_{0} t}(A u, u) d t \\
& -\int_{0}^{\infty} e^{-4 \pi s_{0} t}\left(\int_{0}^{t} a(t-\tau) A u(\tau) d \tau, u\right) d t .
\end{aligned}
$$

By means of $\left(\frac{\partial u}{\partial t}, u\right)=\frac{1}{2} \frac{d}{d t}\|u(., t)\|^{2}$ and using integration by parts, the left side of (3.6) gives

$$
\int_{0}^{+\infty} e^{-4 \pi s_{0} t}\left(\frac{\partial u}{\partial t}, u\right) d t=-\frac{1}{2}\left\|u_{0}\right\|^{2}+2 \pi s_{0} \int_{0}^{\infty} e^{-4 \pi s_{0} t}\|u(., t)\|^{2} d t .
$$


Using formulas (2.1), (2.10) and the fact that the left side is real, we have

$$
\begin{aligned}
& -\mu \int_{0}^{\infty} e^{-4 \pi s_{0} t}(A u, u) d t-\int_{0}^{\infty} e^{-4 \pi s_{0} t}\left(\int_{0}^{t} a(t-\tau) A u(\tau) d \tau, u\right) d t \\
& =-\mu \int_{-\infty}^{+\infty}(A \check{u}, \check{u})\left(s_{0}+i \delta\right) d \delta-\int_{-\infty}^{+\infty} \check{a}\left(s_{o}+i \delta\right)(A \check{u}, \check{u})\left(s_{0}+i \delta\right) d \delta \\
& =-\int_{-\infty}^{+\infty}\left(\mu+\check{a}\left(s_{0}+i \delta\right)\right)(A \check{u}, \check{u})\left(s_{0}+i \delta\right) d \delta \\
& =-\int_{-\infty}^{+\infty}\left(\mu+\operatorname{Re} \check{a}\left(s_{0}+i \delta\right)\right)(A \check{u}, \check{u})\left(s_{0}+i \delta\right) d \delta .
\end{aligned}
$$

Therefore, if the condition (3.5) is satisfied, then the right side of (3.6) is nonpositive, which implies

$$
2 \pi s_{0} \int_{0}^{\infty} e^{-4 \pi s_{0} t}\|u(., t)\|^{2} d t \leq \frac{1}{2}\left\|u_{0}\right\|^{2}
$$

To estimate the Galerkin-wavelet solution, we need the following lemma.

Let us consider the equation

$$
A u(x)=f(x),
$$

where $A$ is the pseudodifferential operator introduced in Section 1.

Lemma 4. Let $\bar{u}$ be the weak solution of equation (3.8) and $\bar{u}_{h}$ be the Galerkinwavelet solution of this equation, that is, $\left(A \bar{u}_{h}, v\right)=(f, v), \forall v \in V_{h}$. Then if $\bar{u} \in$ $H^{q}\left(\mathcal{J}^{n}\right)(q \geq 2 m)$, then

$$
\left\|\bar{u}-\bar{u}_{h}\right\|_{m} \leq \operatorname{ch}^{q-2 m}\|\bar{u}\|_{q},
$$

where $c$ is a constant independent of $u$ and $h$.

Proof. The assumption gives $\left(A\left(\bar{u}-\bar{u}_{h}\right), v\right)=0, \forall v \in V_{h}$. So it is easy to see that Lemma 3 yields

$$
\begin{aligned}
c^{\prime}\left\|\bar{u}-\bar{u}_{h}\right\|_{m}^{2} & \leq\left(A\left(\bar{u}-\bar{u}_{h}\right), \bar{u}-\bar{u}_{h}\right)=\left(A\left(\bar{u}-\bar{u}_{h}\right), \bar{u}-v\right) \\
& =\sum_{\xi \in \mathbb{Z}^{n}} \sigma(\xi) \mathcal{F}\left(\bar{u}-\bar{u}_{h}\right)(\xi) \mathcal{F}(\bar{u}-v)(\xi) \\
& \leq \sum_{\xi \in \mathbb{Z}^{n}}|\sigma(\xi)|\left|\mathcal{F}\left(\bar{u}-\bar{u}_{h}\right)(\xi)\right||\mathcal{F}(\bar{u}-v)(\xi)| \\
& \leq c^{\prime \prime} \sum_{\xi \in \mathbb{Z}^{n}}\langle\xi\rangle^{2 m}\left|\mathcal{F}\left(\bar{u}-\bar{u}_{h}\right)(\xi)\right||\mathcal{F}(\bar{u}-v)(\xi)| \\
& \leq c^{\prime \prime}\left(\sum_{\xi \in \mathbb{Z}^{n}}\langle\xi\rangle^{2 m}\left|\mathcal{F}\left(\bar{u}-\bar{u}_{h}\right)(\xi)\right|^{2}\right)^{\frac{1}{2}}\left(\sum_{\xi \in \mathbb{Z}^{n}}\langle\xi\rangle^{2 m}|\mathcal{F}(\bar{u}-v)(\xi)|^{2}\right)^{\frac{1}{2}} \\
& \leq c^{\prime \prime}\left\|\bar{u}-\bar{u}_{h}\right\|_{m}\|\bar{u}-v\|_{m},
\end{aligned}
$$

$\forall v \in V_{h}$, where $c^{\prime \prime}$ is a constant independent of $u$ and $h$. 
We obtain thus $\left\|\bar{u}-u_{h}\right\|_{m} \leq c\|\bar{u}-v\|_{m}, \forall v \in V_{h}$. Since $v$ is arbitrary in $V_{h}$, we get $\left\|\bar{u}-\bar{u}_{h}\right\|_{m} \leq c \inf _{v \in V_{h}}\|\bar{u}-v\|_{m}$. The desired estimate is followed immediately by Theorem 1

Corollary 1. Let $w:[0, \infty) \rightarrow V_{h}$ be a $C^{1}$-mapping satisfying

$$
\left(A \frac{\partial^{j} w}{\partial t^{j}}, v\right)=\left(A \frac{\partial^{j} u}{\partial t^{j}}, v\right), j=0,1
$$

for all $v \in V_{h}$, with $u$, a weak solution of problem (1.1)-(1.2). If $\frac{\partial^{j} u}{\partial t^{j}} \in H^{q}\left(\mathcal{J}^{n}\right)(j=$ $0,1 ; q \geq 2 m)$ uniformly in $t \in[0, \infty)$, then

$$
\left\|\frac{\partial^{j}(u-w)}{\partial t^{j}}\right\|_{m} \leq C h^{q-2 m}\left\|\frac{\partial^{j} u}{\partial t^{j}}\right\|_{q}, j=0,1,
$$

where $C$ is a constant independent of $u, h$.

Remark 1. Under the assumption of Section 1, the condition (3.10) always holds, because we have

$$
\frac{d}{d t}(A w, v)=\frac{d}{d t}(A u, v), v \in V_{h} .
$$

Theorem 3. Let $u(x, t) \in L_{2}\left(H^{q, s_{0}}\right)$ be a solution of (3.1)-(3.2) and $u_{h}(x, t) a$ solution of (3.3)-(3.4). If (3.5) is satisfied and $\frac{\partial^{j} u}{\partial t^{j}} \in H^{q}\left(\mathcal{J}^{n}\right)(j=0,1 ; q \geq 2 m)$ uniformly in $t \in[0, \infty)$, then

$$
\begin{aligned}
\left\|u-u_{h}\right\|_{L_{2}\left(H^{\left.0, s_{0}\right)}\right.}^{2} \leq C\left\{\left\|u_{0}-u_{0, h}\right\|^{2}+\right. & h^{2(q-2 m)}\left[\left\|u_{o}\right\|_{q}^{2}\right. \\
& \left.\left.+\int_{0}^{\infty} e^{-4 \pi s_{0} t}\left(\|u\|_{q}^{2}+\left\|\frac{\partial u}{\partial t}\right\|_{q}^{2}\right) d t\right]\right\} .
\end{aligned}
$$

Proof. With $w$ defined by (3.10), from (3.1), (3.3) it follows that for every $v \in V_{h}$ we obtain

$$
\begin{aligned}
& \left(\frac{\partial(u-w)}{\partial t}, v\right)=-\mu(A u, v)-\int_{0}^{t} a(t-\tau)(A u(\tau), v) d \tau+(b, v)-\left(\frac{\partial w}{\partial t}, v\right) \\
& =-\mu(A u, v)-\int_{0}^{t} a(t-\tau)(A u(\tau), v) d \tau+\mu\left(A u_{h}, v\right) \\
& \quad+\int_{0}^{t} a(t-\tau)\left(A u_{h}(\tau), v\right) d \tau+\left(\frac{\partial u_{h}}{\partial t}, v\right)-\left(\frac{\partial w}{\partial t}, v\right) \\
& =\mu\left(A\left(u_{h}-u\right), v\right)+\int_{0}^{t} a(t-\tau)\left(A\left(u_{h}-u\right)(\tau), v\right) d \tau+\left(\frac{\partial\left(u_{h}-w\right)}{\partial t}, v\right) \\
& =\mu\left(A\left(u_{h}-w\right), v\right)+\int_{0}^{t} a(t-\tau)\left(A\left(u_{h}-w\right)(\tau), v\right) d \tau+\left(\frac{\partial\left(u_{h}-w\right)}{\partial t}, v\right)
\end{aligned}
$$


Choosing $v=u_{h}-w$, multiplying two sides of the last equality with $e^{-4 \pi s_{0} t}$ and integrating in $t$ from 0 to $+\infty$, we get

$$
\begin{aligned}
& \int_{0}^{\infty} e^{-4 \pi s_{0} t}\left(\frac{\partial(u-w)}{\partial t}, u_{h}-w\right) d t \\
& =\mu \int_{0}^{\infty} e^{-4 \pi s_{0} t}\left(A\left(u_{h}-w\right), u_{h}-w\right) d t+\int_{0}^{\infty} e^{-4 \pi s_{0} t}\left(\frac{\partial\left(u_{h}-w\right)}{\partial t}, u_{h}-w\right) d t \\
& \quad+\int_{0}^{\infty} e^{-4 \pi s_{0} t} \int_{0}^{t} a(t-\tau)\left(A\left(u_{h}-w\right)(\tau), u_{h}-w\right) d \tau d t \\
& =\int_{-\infty}^{+\infty}\left(\mu+\operatorname{Re} \check{a}\left(s_{0}+i \delta\right)\right)\left(A\left(u_{h}-w\right)\left(s_{0}+i \delta\right),\left(u_{h}-w\right)\left(s_{o}+i \delta\right)\right) d \delta \\
& \quad+\int_{0}^{\infty} e^{-4 \pi s_{0} t}\left(\frac{\partial\left(u_{h}-w\right)}{\partial t}, u_{h}-w\right) d t .
\end{aligned}
$$

In view of (3.5) the first term in the last equality is nonnegative, and we thus have

$$
\int_{0}^{\infty} e^{-4 \pi s_{0} t}\left(\frac{\partial\left(u_{h}-w\right)}{\partial t}, u_{h}-w\right) d t \leq \int_{0}^{\infty} e^{-4 \pi s_{0} t}\left(\frac{\partial(u-w)}{\partial t}, u_{h}-w\right) d t .
$$

By integrating by parts, and using the equality $\left(\frac{\partial u}{\partial t}, u\right)=\frac{1}{2} \frac{d}{d t}\|u\|^{2}$, it is easy to see that the left side of (3.13) equals

$$
-\frac{1}{2}\left\|u_{0 h}-w_{0}\right\|^{2}+2 \pi s_{0} \int_{0}^{\infty} e^{-4 \pi s_{0} t}\left\|u_{h}-w\right\|^{2} d t
$$

where $w_{0}=w(x, 0)$. In view of the Cauchy inequality and the inequality $a b \leq$ $\frac{1}{4 \epsilon} a^{2}+\epsilon b^{2}, a, b \geq 0$ for $\epsilon=\pi s_{0}$, the right side of (3.13) is estimated by

$$
\frac{1}{4 \pi s_{0}} \int_{0}^{\infty} e^{-4 \pi s_{0} t}\left\|\frac{\partial(u-w)}{\partial t}\right\|^{2} d t+\pi s_{0} \int_{0}^{\infty} e^{-4 \pi s_{0} t}\left\|u_{h}-w\right\|^{2} d t .
$$

From (3.13), (3.14), (3.15) it follows that

$$
\begin{aligned}
\int_{0}^{\infty} e^{-4 \pi s_{0} t}\left\|u_{h}-w\right\|^{2} d t \leq & \frac{1}{2 \pi s_{0}}\left\|u_{0 h}-w_{0}\right\|^{2} \\
& +\frac{1}{4 \pi^{2} s_{0}^{2}} \int_{0}^{\infty} e^{-4 \pi s_{0} t}\left\|\frac{\partial(u-w)}{\partial t}\right\|^{2} d t .
\end{aligned}
$$

Corollary (11) yields

$$
\begin{gathered}
\left\|u_{0 h}-w\right\|^{2} \leq 2\left\|u_{0 h}-u_{0}\right\|^{2}+2 C h^{2(q-2 m)}\left\|u_{0}\right\|_{q}^{2} \\
\int_{0}^{\infty} e^{-4 \pi s_{0} t}\left\|\frac{\partial(u-w)}{\partial t}\right\|^{2} d t \leq C h^{2(q-2 m)} \int_{0}^{\infty} e^{-4 \pi s_{0} t}\left\|\frac{\partial u}{\partial t}\right\|_{q}^{2} d t
\end{gathered}
$$

By using again Corollary 1 and the inequality $(a+b)^{2} \leq 2 a^{2}+2 b^{2}$, we get

$$
\left\|u-u_{h}\right\|_{L_{2}\left(H^{\left.0, s_{0}\right)}\right.}^{2} \leq C h^{2(q-2 m)}\|u\|_{L_{2}\left(H^{\left.0, s_{0}\right)}\right.}^{2}+2\left\|u_{h}-w\right\|_{L_{2}\left(H^{\left.0, s_{0}\right)}\right.}^{2} .
$$

The desired estimate in the theorem now follows from (3.16), (3.17), (3.18) and (3.19).

\section{ACKNOWLEDGEMENT}

The authors would like to thank the Editor for helpful suggestions by which the paper is improved. 


\section{REFERENCES}

[1] M.V. Altaiski, p-adic wavelet decomposition vs Fourier analysis on spheres, Indian J. pure appl. Math. 1997, 28(2):197-205. MR 98b:42056

[2] G. Beyklin, R. Coifman and V. Rocklin, Fast wavelet transforms and Numerical Algorithms I, Communications on Pure and Applied Mathematics, vol. XLIV (1991) 141-183. MR 92c:65061

[3] Nguyen Minh Chuong, Nguyen Minh Tri and Le Quang Trung, Theory of partial differential equations, Sci. and Techn. Publishing House, Hanoi, 1995. MR 99c:35001

[4] Nguyen Minh Chuong (Chief Author), Nguyen Van Khai, Khuat Van Ninh, Nguyen Van Tuan, and Nguyen Tuong, Numerical Analysis, Education Publishing House, Hanoi, 2001.

[5] Nguyen Minh Chuong, Parabolic pseudodifferential operators of variable order, Dokl. Akad. Nauk SSSR 258(1981), No 6, 1308-1312. MR 83b:35077

[6] Nguyen Minh Chuong, Parabolic pseudodifferential operators of variable order in Sobolev spaces with weighted norms, Dokl. Akad. Nauk SSSR 264(1982), No 2, 299-302. MR 83m:35151

[7] Nguyen Minh Chuong, Degenerate parabolic pseudodifferential operators of variable order, Dokl. Akad. Nauk SSSR 268(1983), No 5, 1055-1058, MR 85g:35068

[8] Nguyen Minh Chuong and Bui Kien Cuong, Galerkin-wavelet approximation for a class of partial integro-differential equations, Fractional Calculus and Applied Analysis, 4(2001), No 2, 143-152. MR 2002c:65246

[9] Nguyen Minh Chuong and Ta Ngoc Tri, The integral wavelet transform in $L_{p}(\mathbb{R}), 1 \leq p \leq \infty$, Fractional Calculus and Applied Analysis, 3(2000), No 2, 133-140. MR 2001k:42035

[10] Nguyen Minh Chuong and Bui Kien Cuong, The convergence estimates for Galerkin-wavelet method for initial pseudodifferential periodic problems, International J. of Math. Sciences, Volume 2003, No 14, 857-867. MR 2004b:35350

[11] Nguyen Minh Chuong (Chief Author), Ha Tien Ngoan, Nguyen Minh Tri, and Le Quang Trung, Partial Differential Equations, Second Edition, Education Publishing House, Hanoi, 2000. MR 2001i:35001

[12] Nguyen Minh Chuong and Nguyen Van Co, The multidimensional p-adic Green Function, Proc. AMS, 127(1999), No 3, 385, 694. MR 99i:11109

[13] Stephan Dahlke and Ilona Weindrich, Wavelet bases Adapted to Pseudodifferential Operators, Applied and Computational Harmonic Analysis, 1(1994), 267-283. MR 96a:35226

[14] W. Dahmen, S. Prössdorf, R. Schneider, Wavelet approximation methods for pseudodiffirential equation I: Stability and convergence, Math. Zeist. Springer-Verlag, 215(1994), 583-620. MR 95g:65148

[15] David L. Donoho, Nonlinear wavelet methods for recovery of signals, densities, and spectra from indirect and noisy data, Proc. Sympos. Appl. Math. 47, AMS, Providence, RI, (1993). MR 95k:62099

[16] Yu. V. Egorov, Nguyen Minh Chuong, On some semilinear boundary value problems for singular integro-differential equations, Russian Math. Surveys, 53(1998), No 6, 1348-1349. MR 2000j:35040

[17] Peter R. Massopust, Fractal functions, fractal surfaces, and wavelets, Academic Press, San Diego, New York, Boston, London, Sydney, Tokyo, Toronto, 1994. MR 96b:28007

[18] Y. Meyer, Ondelettes et Opérators, I, II, Hermann, Paris, 1990, MR 93i:42002 MR 93i:42003

[19] L. Hörmander, The analysis of linear partial operator, III, IV, Springer-Verlag, Berlin Heidelberg New York Tokyo, 1985. MR 88m:35001; MR 89k:35002

[20] V. Strela and G. Strang, Finite element multiwavelets, Approximation Theory and Applications, Maratea, 1994, NATO Adv. Sci. Inst. Ser. C Math. Phys. Sci., 454. MR 96c:42072

[21] M. E. Taylor, Noncommutative Microlocal Analysis, Memoirs of the AMS, part I, Vol. 52, No. 313, AMS Providence, RI, 1984. MR 86f:58156

[22] F. Trèves, Introduction to pseudodifferential and Fourier Integral Operators, I, II, Plenum Press. New York and London, 1982, MR 85d:58077, MR 85d:35110

Hanoi Institute of Mathematics, P.O. Box 631 Bo Ho, 10000 Hanoi, Vietnam

E-mail address: nmchuong@math.ac.vn

Hanoi Institute of Mathematics, P.O. Box 631 Bo Ho, 10000 Hanoi, Vietnam 